\title{
Influence of strain softening on the fracture of plain concrete beams
}

\author{
H. ANANTHAN, B.K. RAGHUPRASAD and K.T. SUNDARA RAJA IYENGAR \\ Department of Civil Engineering, Indian Institute of Science, Bangalore 560 012, India
}

Received 20 August 1988; accepted in revised form 7 June 1989

\begin{abstract}
Fracture behaviour of notched and un-notched plain concrete slender beams subjected to three-point or four-point bending is analyzed through a one-dimensional model, also called Softening Beam Model. Fundamental equations of equilibrium are used to develop the model. The influence of structural size in altering the fracture mode from brittle fracture to plastic collapse is explained through the stress distribution across the uncracked ligament obtained by varying the strain softening modulus. It is found that at the onset of fracture instability, stress at the crack tip is equal to zero. The maximum load and fracture load are found to be different and a unique value for the fracture load is obtained. It is shown that the length of the fracture process zone depends on the value of the strain softening modulus. Theoretical limits for fracture process zone length are also calculated. Several nonlinear fracture parameters, such as, crack tip opening displacement, crack mouth opening displacement and fracture energy are computed for a wide variety of beam specimens reported in the literature and are found to compare very well with experimental and theoretical results. It is demonstrated that by following a simple procedure, both pre-peak and post-peak portions of load versus crack mouth opening displacement curve can be obtained quite accurately. Further, a simple procedure to calculate the maximum load is also developed. The predicted values of maximum load are found to agree well with the experimental values. The Softening Beam Model (SBM), proposed in this investigation is very simple and is based on rational considerations. It can completely describe the fracture process from the beginning of formation of the fracture process zone till the onset of fracture instability.
\end{abstract}

\section{Introduction}

Numerous experimental investigations performed on notched plain concrete beams subjected to three-point or four-point bending have revealed the fracture process to be dependent on size of the structural member. It is generally accepted that, smaller specimens tend to fail by plastic collapse, while the larger specimens fail by brittle fracture. It has been concluded by Carpinteri [1], that, specimen size alone can alter the modes of failure. Bazant and Cedolin $[2,3]$, through numerical approach, have pointed out that with change in structural size a transition from a tensile strength collapse to a crack propagation collapse occurs. The results obtained by Peterson [4], by performing extensive numerical simulations of crack propagation in a three-point bend test show also that, when the structural sizes are increased, the material becomes more and more brittle. Carpinteri [5], has concluded that an embrittlement occurs by increasing the structural size, a fact which corresponds to the fundamental laws of physical similitude.

Several theoretical models have been proposed to explain the fracture process as well as size dependency in concrete. Hillerborg et al. [6], have proposed a "fictitious crack model" also known as a "damage zone model". The tensile stress is not assumed to fall to zero immediately after the attainment of limiting value but to decrease with increasing crack 
widths. Modulus of elasticity, $E$, uniaxial tensile strength, $\sigma_{t}$, and fracture energy, $G_{F}$, defined as the area under post-peak stress versus the crack opening displacement diagram are the material properties required to describe the tensile fracture behaviour of concrete.

Bazant and Oh [7] introduced the concept of "crack band theory" for fracture of concrete. The fracture front is modelled as a blunt smeared crack band. The material fracture properties are characterized by three parameters $G_{F}, \sigma_{t}$ and the width of crack band, $w_{c}$ (fracture process zone). $G_{F}$ is, however, defined as the product of $w_{c}$ and the area under the tensile stress-strain curve.

Nallathambi and Karihaloo [8] modified the fictitious crack model by accounting for the nonlinearity in the stress-strain plot prior to the attainment of peak by reducing the value of $E$. The load-deflection plot obtained is reported to be relatively closer to the experimental one.

The models mentioned above, irrespective of the approach adopted, require a complete stress-crack opening relation. They are particularly well suited for numerical techniques like the finite element method. Several objections have been raised [8] towards the use of $G_{F}$, considered as a possible reliable indicator of fracture. It is found to be extremely size dependent. Further it is also very tedious to determine it by performing a stable tension test. Uncertainty exists with the true fracture area, owing to the tortuous nature of crack propagation in concrete.

Sih [9] and Carpinteri [10] have used the concepts of strain energy density theory and strain softening behaviour to simulate numerically the fracture behaviour of concrete.

Some models which do not require the finite element technique are also proposed. Wecharatana and Shah [11], based on some simple and approximate extensions of the concepts of linear elastic fracture mechanics, have predicted the extent of a nonlinear fracture process zone in concrete. Critical crack opening displacement equal to $0.025 \mathrm{~mm}$ and a constant closing pressure to exist along the length of the fracture process zone are assumed. Fracture loads of a large number of notched beams are reported to have been estimated with a reasonable degree of accuracy.

Kobayashi et al. [12] have demonstrated through their model that by assuming the closing pressure distribution to vary linearly from zero at the crack tip to $\sigma_{t}$ and then to remain constant, a better agreement with respect to load versus crack mouth displacement curve can be obtained. The length of the unloading zone was chosen such that the crack tip opening displacements at the macrocrack tip and at the beginning of the unloading zone were 0.038 and $0.0127 \mathrm{~mm}$, respectively.

In these models constant values for crack opening displacement and arbitrary closing pressure distribution are assumed. However critical crack opening displacement is found to vary with specimen size.

Jenq and Shah [13] have proposed a "two parameter fracture model". The two parameters are the critical stress intensity factor calculated at the tip of the effective crack and the elastic critical crack opening displacement. Based on their test results the two parameters are found to be size independent. In contrast to modulus of rupture, tensile strength of unnotched concrete specimens is demonstrated to be size independent. The validity of the values for the two parameters even in the cases of beams possessing different span to beam depth ratios is yet to be established.

Some nondimensional numbers also known as brittleness numbers have been proposed by Carpinteri [5], Hillerborg [6], Jenq and Shah [13], and Bazant and Pfeiffer [14] to characterize the effect of structural size $(W)$ on the fracture behaviour of concrete beam specimens. 
Parameters like $K_{c}$ or $G_{F}, \sigma_{i}, E_{c}$ and $W$ are considered for this purpose. However these brittleness numbers cannot be directly determined owing to the nonavailability of unique values for $K_{c}$ or $G_{F}$. The influence of structural size on the collapse stress can be quantified only when the values for brittleness numbers are assumed, otherwise only qualitative predictions can be made. Hence size effects are not yet quantitatively described.

In this paper a one-dimensional model based on fundamental equations of equilibrium is developed to explain the fracture behaviour of notched and un-notched plain concrete beams subjected to three-point or four-point bending. The influence of structural size in altering the failure mode is explained through the stress distribution obtained across the uncracked ligament. The key factor affecting the stress distribution is found to be the strain softening modulus and is considered to be dependent on the structural size (uncracked ligament). It is shown that at the onset of fracture instability, stress at the crack tip should be equal to zero and distinction to exist between maximum load and fracture load is clearly established. The performance of the model is evaluated in the light of experimental and numerical results obtained by various investigators in the case of notched plain concrete beams, with regard to several nonlinear fracture parameters, such as, nonlinear process zone length, critical crack opening displacement, load versus crack mouth opening displacement curves and $G_{F}$. It is demonstrated that the model can predict quite accurately the maximum load of a wide variety of beam specimens reported in the literature. Some of the theoretical aspects adopted in the present model are addressed in the following sections.

\section{Softening behaviour of concrete in tension}

The direct tensile tests performed by various investigators [15-19] have shown that the overall load-elongation curve presents a peak followed by a softening branch. Based on these tests, it has been asserted that before global rupture, a fracture process zone (damage zone) forms locally. The material in the fracture process zone softens, while the material in the undamaged portion still behaves in a proportional manner. Hillerborg et al. [6], have assumed the behaviour of tensile fracture zone to be a material property. Carpinteri $[10,20]$ has explained the size effects on the descending branch of the stress-strain curve which appears to vary with specimen size. He has pointed out that, similarity between physical fracture behaviour of the specimen (global) and the behaviour of the fracture process zone (local) can exist only if the fracture process zone length is proportional to the size of the structural member. However, with increasing size of the specimens, though softening in the fracture process zone can take place by the same extent, physical fracture behaviour of the specimen can proceed in a totally different manner. It has been summarized by Carpinteri [21] that by increasing the nonlinearity of the material, a transition in the fracture behaviour observed with decreasing structural sizes can be achieved. It has been fairly well established $[15,19]$ that unique post-peak stress versus strain or deformation diagram does not exist in concrete. In the present model, the strain softening modulus is considered to be a structural property.

\section{Structural behaviour of plain concrete beams}

As plain concrete is relatively weak in tension, the ultimate strength of such a beam primarily depends on the limiting tensile strength of concrete and the nature of its distribution across 


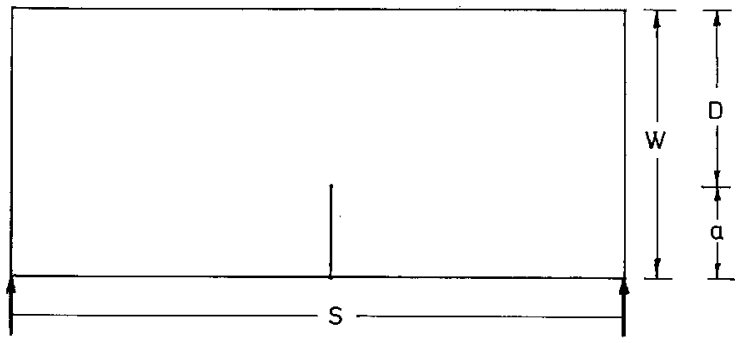

(a)

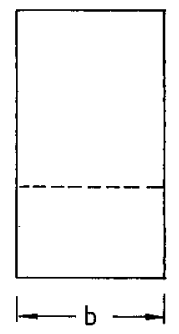

(b)

Fig. 1. Beam nomenclature, (a) Longitudinal section of the beam, (b) Cross-section at mid-span.

the uncracked ligament. Since the stress-strain curve is influenced by the structural size, a unique constitutive law describing the stress variations with increasing strains especially in the post peak region cannot exist. Figure 1(a) shows the longitudinal section of the beam and 1 (b) the cross-section at mid span. Considering the two extreme idealizations for the stress-strain curve indicated by the lines $E^{*}=\infty$ and $E^{*}=0$ (Fig. 2(a)), $E^{*}$ being the ratio of strain softening modulus, $E_{T}$, to initial modulus of elasticity, $E$, stress distributions obtained across the uncracked ligament are respectively as in Figs. 3(a) and 3(b). Assuming that, the maximum moment carrying capacity of the beam is governed only by the net section strength of the uncracked ligament and for the stress distribution of the type shown in Fig. 3(a), $M_{\max }=\sigma_{t} b D^{2} / 6$ is obtained. Denoting $\sigma_{t} b D^{2} / 6$ as $M_{n}$, the value of the ratio $M_{\max } / M_{n}$, equal to unity, for $E^{*}=\infty$, is obtained. Similarly with reference to Fig. 3(b) and in the limit $\alpha$ and $\beta$ tending to zero $M_{\max } / M_{n}$ equal to three, for $E^{*}=0$, is obtained. $M_{\max } / M_{n}=1$, represents brittle fracture and that equal to 3 , represents plastic collapse. The assumptions involved in obtaining these values of $M_{\max } / M_{n}$ are that, concrete in tension behaves as a linear elastic perfectly brittle or plastic material, while in compression it still behaves in a linearly proportional manner, which is justifiable with reference to Fig. 3(a). However, with Fig. 3(b), this requires concrete to possess an infinite compressive strength. Hence the value of $M_{\max } / M_{n}$ equal to three can only be treated as a hypothetical theoretical limit on the maximum value of $M_{\max } / M_{n}$, attainable by a section of the beam. Different values of $M_{\max } / M_{n}$ in the range 1 to 3 can be obtained with different stress distribution diagrams of the type shown in Fig. 3(c), in which the value of $E^{*}$ will lie between infinity and zero, thus representing the transition in fracture behaviour. From the above reasoning, it can be concluded that the value of the ratio $M_{\max } / M_{n}$ can serve as an indicator for characterizing the type of fracture process. Further, the ratio can be determined very easily from experimental results. Basic concepts of the proposed model along with mathematical expressions are outlined in the following sections.

\section{Proposed model}

Since concrete is generally known to exhibit strain softening in tension, brittle fracture or perfect plastic collapse seem to be only idealizations. Hence by modelling concrete according to the strain softening behaviour and by varying the strain softening slope between infinity to zero a transition between the two extreme idealizations can be achieved. Since $M_{\max } / M_{n}$ 


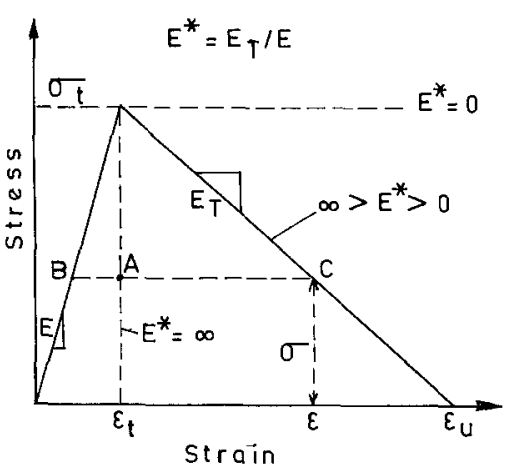

(a)

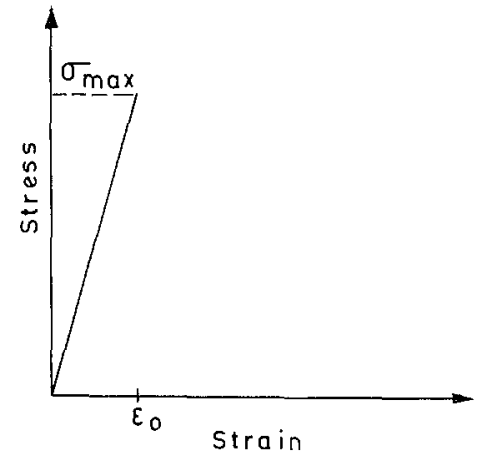

(b)

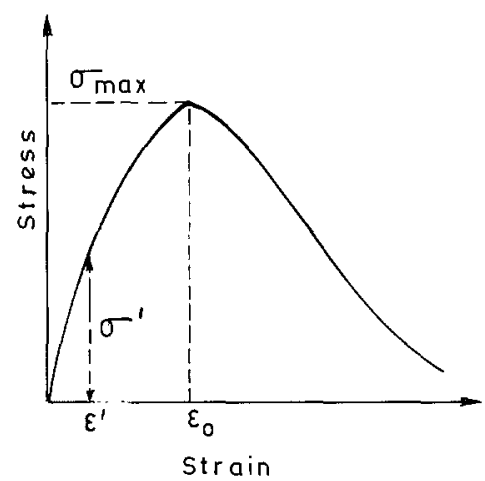

(c)

Fig. 2. Constitutive laws for concrete, (a) stress-strain diagram in tension, (b) and (c) are respectively linear and nonlinear stress-strain diagrams in compression.

can characterize the type of fracture of the beam of given dimensions, it becomes necessary to determine the stress block across the uncracked ligament.

The stress-strain diagram for concrete in tension can be approximated to be bilinear as shown in Fig. 2(a). The stress $\sigma$ in the strain softening range can be expressed as,

$$
\sigma=\sigma_{t}-E_{T}\left(\varepsilon-\varepsilon_{t}\right)
$$

in which $\sigma_{t}=$ limiting uniaxial tensile strength, $E_{T}=$ strain softening modulus, $\varepsilon, \varepsilon_{t}=$ tensile strain corresponding to $\sigma$ and $\sigma_{t}$ respectively.

With the additional assumptions regarding compressive stresses to vary linearly (Fig. 2(b)) with increasing strains (being justified in Section 4.3), and the strain distribution to possess linear variation across the uncracked ligament, stress and strain distribution obtained across the uncracked ligament are shown respectively in Figs. 3(c) and 3(d). The assumption that plane section remains plane even after deformation is usually made in the derivation of beam theory. This assumption is valid for slender beams as shear deformation effects are negligible and is certainly not valid for deep beams. Regarding the validity of this assumption in the case of beams with notches or cracks it is worth mentioning that a large number of tests on reinforced concrete members have indicated that this assumption is very nearly correct at all 


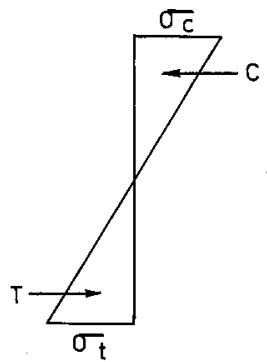

(a)

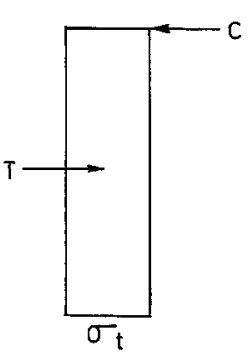

(b)

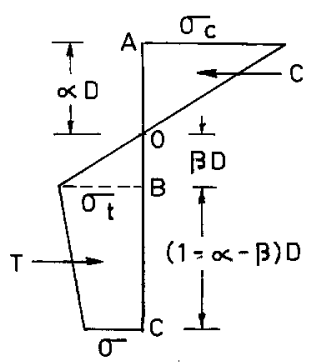

(c)

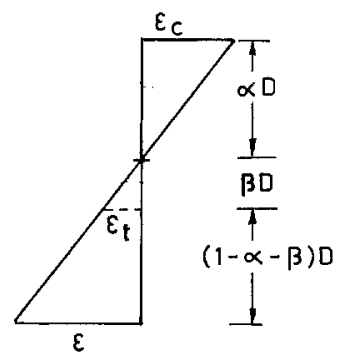

(d)

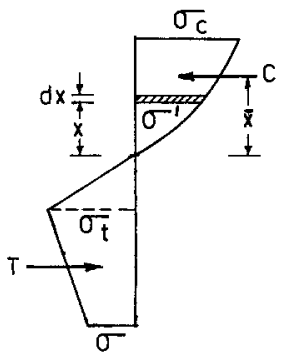

(e)

Fig. 3. Stress and strain distributions (a), (b) and (c) are respectively the stress blocks for $E^{*}=\infty, E^{*}=0$ and $\infty>E^{*}>0$, (d) strain diagram, (e) stress block for $\infty>E^{*}>0$.

stages of loading up to flexural failure. Certainly it is accurate in the compression zone of the concrete. This assumption may not be completely valid in the immediate neighbourhood of a crack. However if the concrete strain is measured over a gauge length that includes cracks, it is found that this assumption is valid. Since in this work attention is focussed upon mode I type of fracture of slender beams only, this assumption seems to be very reasonable and by virtue of which the model is considerably simplified.

$\varepsilon$ can be expressed as (Fig. 3(d)).

$$
\varepsilon=\frac{1-\alpha}{\beta} \varepsilon_{t}
$$

Substituting for $\varepsilon$ as given by (4.2) in (4.1), one obtains

$$
\sigma=\sigma_{t}-\frac{E_{T} \varepsilon_{t}(1-\alpha-\beta)}{\beta}
$$

Replacing $\varepsilon_{t}$ by $\sigma_{t} / E$, and denoting $E_{T} / E$ as $E^{*},(4.3)$ can be written as

$$
\sigma=\sigma_{i}\left[1-E^{*} \frac{(1-\alpha-\beta)}{\beta}\right]
$$


The maximum compressive stress $\sigma_{c}$ can be expressed as (Fig. 3(c))

$$
\sigma_{c}=\frac{\alpha}{\beta} \sigma_{t}
$$

Thus with a knowledge of $\alpha, \beta$ and $E_{T}$, the stress block across the uncracked ligament can be completely characterized through (4.4) and (4.5). Since the cracked section is in equilibrium, fundamental equations of equilibrium have to be satisfied up to the onset of fracture. As the cracked section is not subjected to any externally applied horizontal force, the total compressive force shall be equal to the total tensile force. Further, the moment of resistance developed by the net section shall be equal to the external bending moment.

Referring to Fig. 3(c), and invoking the first condition of equilibrium,

$$
\frac{1}{2} \sigma_{c} \alpha D b=\frac{1}{2} \sigma_{i} \beta D b+\frac{1}{2}\left(\sigma_{t}+\sigma\right)(1-\alpha-\beta) D b
$$

is obtained.

Substituting for $\sigma$ and $\sigma_{c}$ according to (4.4) and (4.5), (4.6) after simplification can be written as

$$
\alpha^{2}\left(1+E^{*}\right)+2 \alpha\left(\beta+E^{*} \beta-E^{*}\right)+\left[\beta^{2}\left(1+E^{*}\right)-2 \beta\left(1+E^{*}\right)+E^{*}\right]=0
$$

The roots of the above quadratic equation are given by

$$
\alpha=\frac{-\left(\beta+E^{*} \beta-E^{*}\right) \pm \sqrt{2 \beta E^{*}+2 \beta-E^{*}}}{\left(1+E^{*}\right)}
$$

According to moment equilibrium condition, if $M$ is the external bending moment acting on the cross-section, then moment of the tensile forces about the centre of compression can be written as

$$
\begin{aligned}
M= & \frac{b D^{2}}{6}\left[2 \sigma_{1} \beta(\alpha+\beta)+\sigma(1-\alpha-\beta)(\alpha+3 \beta+3)\right. \\
& \left.+\left(\sigma_{t}-\sigma\right)(1-\alpha-\beta)(\alpha+2 \beta+1)\right]
\end{aligned}
$$

Substituting for $\sigma$ according to (4.4), (4.9) after simplification can be obtained in the following non-dimensional form.

$$
\frac{M}{M_{n}}=3-2 \alpha+E^{*} \frac{(\beta+2)}{\beta}(2 \beta+2 \alpha-1)-(\alpha+\beta)^{2}\left[1+\frac{E^{*}(\beta+2)}{\beta}\right]
$$

It can be easily verified from (4.10), that for $E^{*}=\infty, \beta=\alpha=0.5, M / M_{n}$ equal to unity is obtained. Likewise for $E^{*}=0, \alpha=\beta=0, M / M_{n}$ equal to three results. 


\subsection{Solution procedure}

Only two equations (4.8 and 4.10) are available to determine three unknown parameters $\alpha$, $\beta$ and $E^{*}$, required to completely characterize the stress block across the uncracked ligament. Thus, the solution in a straightforward manner seems to be impossible. However, by assuming values for $E^{*}$ and $\beta, \alpha$ can be obtained according to (4.8) and hence $M / M_{n}$ according to (4.10). Since arbitrary values are obtained with this approach, it becomes necessary to check for their physical admissibility. With this in view, the following additional conditions are developed.

Condition 1: For any given value of $E^{*}$, the minimum value of $\beta$ shall be such that the quantity inside the radical sign in (4.8), that is, $\sqrt{2 \beta E^{*}+2 \beta-E^{*}}$ should be equal to zero, for $\alpha$ to be positive and real. This condition is used to get an initial admissible value for $\alpha$, for an assumed value of $\beta$, and to eliminate the imaginary roots of the quadratic equation.

Condition 2: Since two distinct values for $\alpha$ are obtained according to (4.8) for various values of $\beta$ and $E^{*}$, the physically admissible value for $\alpha$, is the one which is not only positive but also yields $M / M_{n}$ obtained from (4.10) as a positive quantity.

Condition 3: The value of $\alpha$ and $\beta$ shall be such that $(\alpha+\beta)$ cannot be greater than unity.

Condition 4: Experimental studies [15-19] reveal that corresponding to any stress level, pre-peak strain is less than post-peak strain as measured from the peak, i.e., $A B<A C$ in Fig. 2(a). With this assumption, maximum value of $E^{*}$ is fixed to be unity, with the minimum value of $E^{*}$ equal to zero. The validity of this condition depends on the length of the tensile specimen. If it is very large it seems to be possible that a softening branch with a positive slope can result. In such cases this condition is not valid.

With the conditions 1 to 4 , operating on the values of $\alpha, \beta$ and $E^{*}$, the following solution procedure is proposed.

1. For any assumed value of $E^{*}$ in the range fixed by Condition 4 , the minimum value of $\beta$ is obtained according to Condition 1 . The corresponding value of $\alpha$ is evaluated from (4.8).

2. This value of $\alpha$ is checked for its admissibility according to Conditions 2 and 3. The corresponding value of $M / M_{n}$ is obtained from (4.10).

3. The minimum value of $\beta$ is then incremented by a small amount and the whole procedure is repeated till a limiting stage according to Condition 3 is reached.

With this solution procedure some of the important results obtained are discussed below

\subsection{Discussion of results}

Variation of $M / M_{n}$ with $\beta$ : The variation of $M / M_{n}$ for various values of $\beta$, for some of the representative values of $E^{*}$ in the range zero to unity is shown in Fig. 4. From this figure it can be seen that $M / M_{n}$ is found to increase from unity corresponding to a maximum value of $\beta$, attaining a maximum value at some intermediate value of $\beta$ and then showing a decreasing trend with a corresponding reduction in the value of $\beta$. This variation is found for all values of $E^{*}$ other than zero. However with $E^{*}=0, M / M_{n}$ is found to increase continuously with a corresponding reduction in the value of $\beta$ and attains a maximum value 


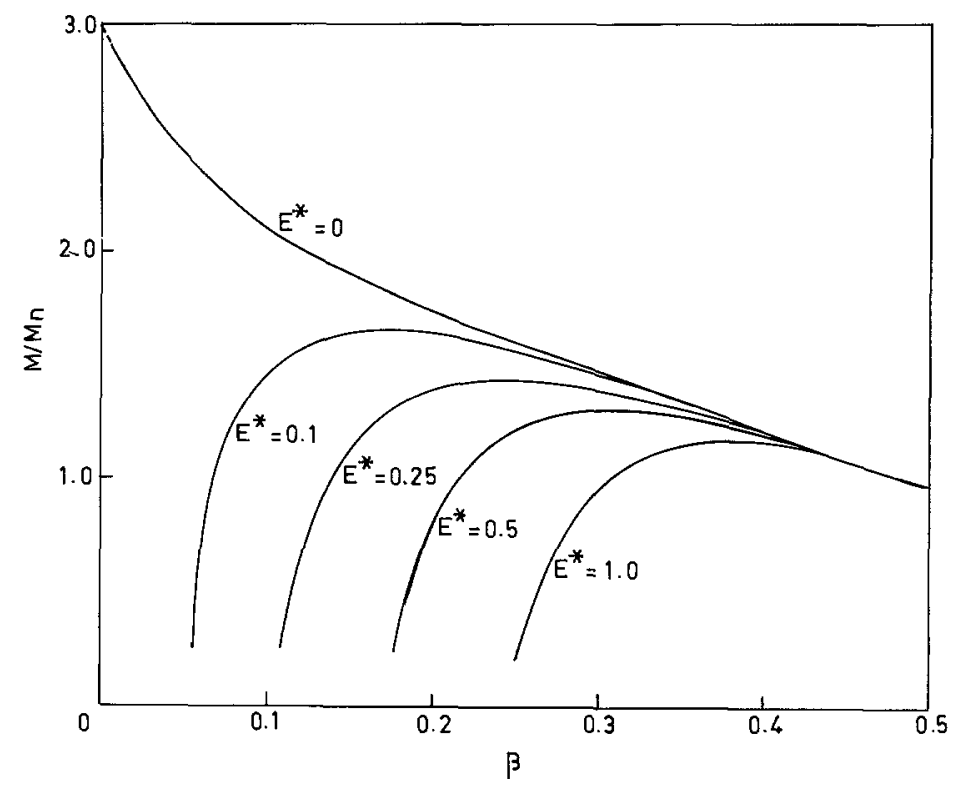

Fig. 4. $M / M_{n}$ versus $\beta$ for some values of $E^{*}$.

corresponding to a minimum value of $\beta$. Similar variation of $\alpha / \beta$ for various values of $\beta$ is found.

Variation of $M_{\max } / M_{n}$ with $E^{*}: M_{\max } / M_{n}$ values obtained for various values of $E^{*}$ in the range $0-1$ is shown in Fig. 5. Increase in the value of $E^{*}$ causes a corresponding decrease in the $M_{\max } / M_{n}$ value. Even a small increase in the value of $E^{*}$ from zero results in a steep decrease in the value of $M_{\max } / M_{n}$. However, this rate of decrease of $M_{\max } / M_{n}$ reduces for increasing values of $E^{*}$ and proceeds with much slower rate as $E^{*}$ approaches unity and thereafter.

Variation of $M / M_{n}$ with $\alpha / \beta$ : The variation of $M / M_{n}$ with $\alpha / \beta$ for some of the representative values of $E^{*}$ in the range zero to unity is shown in Fig. 6. From this figure it can be seen that, both $M / M_{n}$ and $\alpha / \beta$ increase from unity, till $M / M_{n}$ reaches a maximum value $M_{\max } / M_{n}$. Beyond this stage, $\alpha / \beta$ increases and attains a maximum value with a corresponding reduction in the value of $M / M_{n}$. Further, $\alpha / \beta$ decreases with $M / M_{n}$. This interesting variation is found for all values of $E^{*}$ other than zero. However for $E^{*}=0$, both $M / M_{n}$ and $\alpha / \beta$ are found to increase continuously.

\subsection{Effect of nonlinear stress-strain law in compression}

The stress-strain relationship for concrete in compression can be written as [22] (Fig. 2(c))

$$
\sigma^{\prime}=\frac{E \varepsilon^{\prime}}{1+\left(\frac{\varepsilon^{\prime}}{\varepsilon_{0}}\right)^{2}}
$$




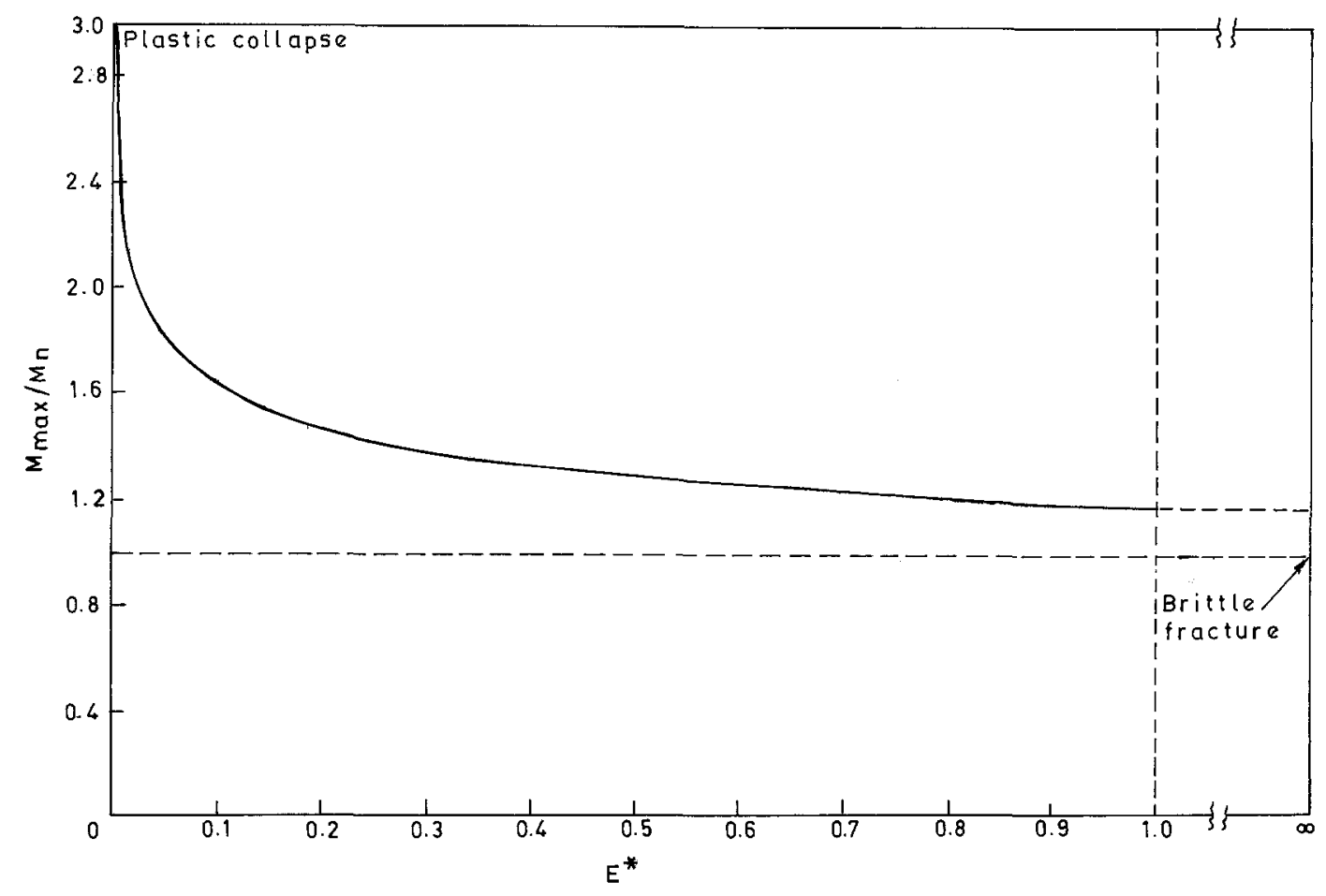

Fig. 5. $M_{\max } / M_{n}$ for all values of $E^{*}$.

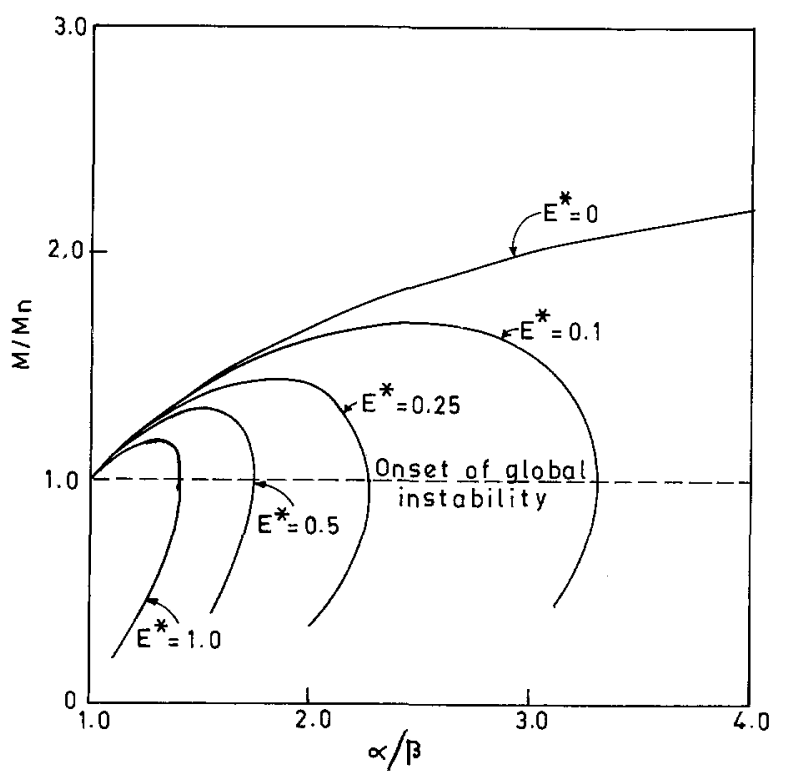

Fig. 6. $M / M_{n}$ versus $\alpha / \beta$, for some values of $E^{*}$.

in which $E=$ initial tangent modulus $=2 \sigma_{\max } / \varepsilon_{0}, \varepsilon_{0}=$ strain corresponding to peak stress $\sigma_{\max }, \varepsilon^{\prime}=$ strain corresponding to the stress $\sigma^{\prime}$.

The stress distribution obtained across the uncracked ligament is as shown in Fig. 3(e).

The total compressive force is obtained as

$C=\int_{0}^{\alpha D} \sigma^{\prime} b \mathrm{~d} x$ 
Substituting for $\varepsilon^{\prime}=x \varepsilon_{t} / \beta D$ in (4.11), after performing the integration and substituting the limits, the following equation for $C$ results

$$
C=\frac{b E \beta D \varepsilon_{0}^{2}}{2 \varepsilon_{i}}\left[\ln \left(1+\left(\frac{\alpha \varepsilon_{i}}{\beta \varepsilon_{0}}\right)^{2}\right)\right]
$$

According to the force-equilibrium condition, the following relationship results.

$$
\frac{b E \beta D \varepsilon_{0}^{2}}{2 \varepsilon_{t}}\left[\ln \left(1+\left(\frac{\alpha \varepsilon_{t}}{\beta \varepsilon_{0}}\right)^{2}\right)\right]=\frac{1}{2} \sigma_{t} D b\left[\beta+2(1-\alpha-\beta)-\frac{E^{*}(1-\alpha-\beta)^{2}}{\beta}\right]
$$

Substituting $\sigma_{t}=E \varepsilon_{t}$, in (4.14), and after rearranging, the following equation is obtained

$$
\begin{aligned}
& \alpha^{2} E^{*}+2 \alpha\left(\beta+\beta E^{*}-E^{*}\right)+\beta^{2}\left[1+E^{*}+\frac{\varepsilon_{0}^{2}}{\varepsilon_{t}^{2}} \ln \left(1+\left(\frac{\alpha \varepsilon_{t}}{\beta \varepsilon_{0}}\right)^{2}\right)\right] \\
& -2 \beta\left(1+E^{*}\right)+E^{*}=0
\end{aligned}
$$

The ratio $\left(\alpha \varepsilon_{t} / \beta \varepsilon_{0}\right)^{2}$ which appears in (4.15) will be less than unity for all values of $\alpha / \beta$ which depend on $E^{*}$ which are possible in a real plain concrete beam. For example, for $\varepsilon_{t}=0.0001$ and $\varepsilon_{0}=0.002$, which are standard peak values in tension and compression respectively, the ratio $\alpha \varepsilon_{t} / \beta \varepsilon_{0}$ will be less than or equal to unity for values of $\alpha / \beta$ less than or equal to 20 . The ratio $\alpha / \beta=20$ implies an extreme compressive fibre strain of $20 \varepsilon_{t}$ (Fig. 3(d)), which is impractically large in a plain concrete beam. Therefore, it may be concluded that for all practical considerations $\alpha / \beta$ will be very much less than 20 and thus $\alpha \varepsilon_{t} / \beta \varepsilon_{0}$ will be very much less than unity. Expanding the logarithmic quantity in (4.15), in the form of series and retaining only the first term of the series expansion, one obtains

$$
\alpha^{2}\left(1+E^{*}\right)+2 \alpha\left(\beta+\beta E^{*}-E^{*}\right)+\beta^{2}\left(1+E^{*}\right)-2 \beta\left(1+E^{*}\right)+E^{*}=0
$$

Equation (4.16), is exactly of the same form as (4.7) obtained by considering a linear relationship between the compressive stress and strain.

The centre of compression is evaluated as,

$$
\bar{x}=\frac{\int_{0}^{\alpha D} \sigma^{\prime} x \mathrm{~d} x}{\int_{0}^{x D} \sigma^{\prime} \mathrm{d} x}
$$

in which $x$ and $\bar{x}$ are measured from the position of the neutral axis.

Performing the integration and substituting the limits, (4.17) can be written as

$$
\bar{x}=\frac{2 \varepsilon_{t}^{2}\left[\frac{\alpha D}{Q}-\frac{\tan ^{-1}(\alpha D \sqrt{Q})}{Q^{3 / 2}}\right]}{\left(\beta D \varepsilon_{0}\right)^{2} \ln \left(1+Q \alpha^{2} D^{2}\right)}
$$


where $Q=\left(\varepsilon_{t} / \beta D \varepsilon_{0}\right)^{2}$.

Using the moment equilibrium condition, $M / M_{n}$, after simplification is obtained as

$$
\begin{aligned}
\frac{M}{M_{n}}= & 3-6 \alpha+3 \alpha^{2}-\beta^{2}-E^{*} \frac{(1-\alpha-\beta)^{2}}{\beta}(2-2 \alpha+\beta) \\
& +\frac{6}{\ln \left(1+\left(\frac{\alpha \varepsilon_{t}}{\beta \varepsilon_{0}}\right)^{2}\right)}\left[-2 \alpha^{2}+2 \alpha-\alpha \beta-E^{*} \frac{\alpha}{\beta}(1-\alpha-\beta)^{2}\right. \\
& \left.+\frac{\varepsilon_{0}}{\varepsilon_{t}} \tan ^{-1}\left(\frac{\alpha \varepsilon_{t}}{\beta \varepsilon_{0}}\right)\left\{E^{*}(\alpha-1)^{2}+\left(1+E^{*}\right)\left(\beta^{2}+2 \alpha \beta-2 \beta\right)\right\}\right]
\end{aligned}
$$

Retaining the first term of the logarithmic series and the first two terms of the inverse tangent series, (4.19) simplifies to

$$
\frac{M}{M_{n}}=3-2 \alpha+E^{*} \frac{(\beta+2)}{\beta}(2 \alpha+2 \beta-1)-(\alpha+\beta)^{2}\left(1+E^{*} \frac{(\beta+2)}{\beta}\right)
$$

Equation (4.20) is of exactly the same form as (4.10), obtained by considering a linear variation between compressive stress and strain. Hence the assumption of linear variation of compressive stresses with increasing strains is very reasonable.

\subsection{Onset of fracture instability}

In the present investigation fracture instability is defined as the stage at which real crack tip propagation occurs. This can be expected to occur only when the process zone develops into a real crack. Once a real crack forms, stresses cannot be transferred across them. This implies that for real crack tip propagation, stress at the crack tip after attaining the peak value should eventually reduce to zero. However, it is not known whether this condition will ensure fracture instability. This question is examined further.

Beam of given cross-sectional dimension and material property has a certain value of $M_{n}$. Any increase or decrease in the $M / M_{n}$ value obviously necessitates a corresponding increase or decrease in the applied load. The ratio $\alpha / \beta$ represents the variation of compressive stress or strain at the topmost fibre of the beam. The observed variation of $M / M_{n}$ with $\alpha / \beta$, (Fig. 6), can also be interpreted as follows:-

Compressive stress or strain continues to increase with load $P$, till $P$ reaches a maximum value $P_{\max }$. Further increase in compressive stress or strain till the attainment of maximum value results in a reduction in $P$. Beyond this stage compressive stress or strain reduces with a corresponding reduction in $P$. This interesting variation of compressive strain with $P$, is found to follow the same trend recorded experimentally by Hillsdorf [23].

Hence the stage corresponding to the maximum value of $\alpha / \beta$ can be considered as the limiting stage of stable equilibrium when the onset of fracture instability can be expected to occur, provided the necessary condition (i.e. $\sigma$ at the crack tip $=0$ ) for crack propagation is satisfied. In order to verify this, the stress at the crack tip has to be evaluated according 
to (4.4), in which $\alpha$ and $\beta$ shall be such that $\alpha / \beta$ is maximum. The procedure to determine the values of $\alpha$ and $\beta$, corresponding to a given value of $E^{*}$, so that $\alpha / \beta$ is maximum is demonstrated in the following section.

\subsubsection{Condition for fracture instability}

From (4.8) and by using the principle of extremum values, the following relationships for the determination of the values of $\alpha$ and $\beta$, such that the ratio $\alpha / \beta$ will be maximum, are obtained

$$
\begin{aligned}
& \alpha=-E^{*}+\sqrt{E^{*}\left(1+E^{*}\right)} \\
& \beta=\frac{E^{*}\left(1+E^{*}\right)-E^{*} \sqrt{E^{*}\left(1+E^{*}\right)}}{\left(1+E^{*}\right)}
\end{aligned}
$$

Substituting the values of $\alpha$ and $\beta$ according to (4.21) and (4.22) in (4.4), stress at the crack tip is obtained as,

$$
\sigma=\sigma_{t}\left[1-\frac{E^{*}}{E^{*}}\right]
$$

It is evident from the above equation, that $\sigma$ reduces to zero for all values of $E^{*}$ other than zero.

Hence from the discussion given above, it can be concluded that the essential condition that the stress at the crack tip equal to zero is also the condition for crack propagation to occur in an unstable manner.

4.4.2. $M / M_{n}$ value corresponding to maximum value of $\alpha / \beta$

$M / M_{n}$ value at any stage can be evaluated provided the stress block corresponding to that stage is completely characterized.

The stress distribution across the uncracked ligament is shown in Fig. 7(a).

According to the moment equilibrium condition, the following relationship is obtained.

$$
M=\frac{1}{2} \sigma_{t} \beta D b\left[\frac{2}{3} \alpha D+\frac{2}{3} \beta D\right]+\frac{1}{2} \sigma_{t}(1-\alpha-\beta) D b\left[\frac{2}{3} \alpha D+\beta D+\frac{D-\alpha D-\beta D}{3}\right]
$$

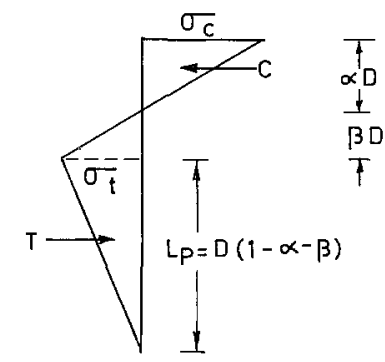

(a)

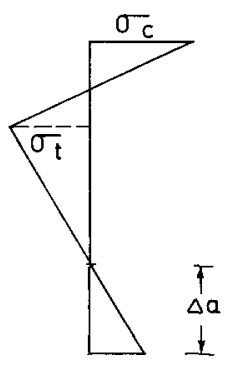

(b)

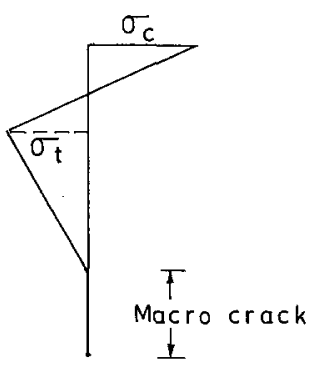

(c)

Fig. 7. Stress diagrams, (a) at the onset of fracture instability, post-peak $M / M_{n}$ equal to unity (b) beyond fracture instability, post-peak $M / M_{n}$ less than unity and (c) corrected for macro crack growth. 
Substituting for $\alpha$ and $\beta$ according to (4.21) and (4.22), (4.24) after simplification, yields $M / M_{n}=1$. From the above discussion, it is clearly established that fracture of plain concrete beams occurs at post-peak value of $M / M_{n}$ equal to unity. In other words in terms of $P$, it is concluded that, fracture of plain concrete beams occurs in the post-peak range at a reduced value of $P$ and not at $P_{\max }$ itself, thus clearly a distinction is drawn between the fracture load and maximum load of beam specimens.

\section{Nonlinear fracture parameters}

Having traced the sequential developments of the proposed model in the preceding sections, its performance for practical applications is evaluated in the light of several experimental and numerical results available in the case of notched plain concrete beams with regard to several nonlinear fracture parameters, such as, nonlinear process zone length, crack tip opening displacement, crack mouth opening displacement and fracture energy. The implementation of the model and the computational procedure adopted in the evaluation of nonlinear fracture parameters are demonstrated in the following sections.

\subsection{Length of fracture process zone}

Fracture process zone is defined as that part of the beam in the tension zone where softening occurs. For initial load applications, till stress at the crack tip attains $\sigma_{t}$, the length of this zone is zero. Beyond this stage, the process zone starts to develop and attains a maximum length prior to fracture. Referring to Fig. 7(a) the length of this zone is given by,

$$
L_{p}=(1-\alpha-\beta) D
$$

Substituting for $\alpha$ and $\beta$ according to (4.21) and (4.22), (5.1) after simplification leads to the following equation.

$$
\frac{E^{*}}{E^{*}+1}=\left[1-\frac{L_{p_{\max }}}{D}\right]^{2}
$$

Denoting $1-\left(L_{p_{\max }} / D\right)$ as $\Delta,(5.2)$ assumes the form

$$
E^{*}=\frac{\Delta^{2}}{1-\Delta^{2}}
$$

For $\Delta=1$ in (5.3), $E^{*}$ tends to infinity, indicating a brittle fracture behaviour and from the definition of $\Delta,\left(L_{p}\right)_{\max }=0$ is implied. Likewise perfect plastic collapse is indicated when $\Delta=0$, for which $E^{*}=0$ and $L_{p_{\max }}=D$. Similarly with $E^{*}=1, \Delta=0.707$ and $L_{p_{\max }}=0.293 D$ are obtained. This indicates the transition in the fracture behaviour. Hence it is concluded that the type of fracture behaviour is well revealed through the extent of formation of the nonlinear fracture process zone, prior to fracture. The key factor controlling $L_{p_{\max }}$ is $E^{*}$. For values of $E^{*}$ in the range 1 to zero considered in the present work, $L_{p_{\max }}$ 
is found to vary between $0.293 D$ to $D$, thus theoretical lower and upper limits for $L_{p_{\max }}$ are clearly established.

The procedure to calculate $L_{p_{\max }}$ is straightforward provided the value of $E^{*}$ is known. In practical situations, $E^{*}$ is an unknown quantity. However, $E^{*}$ can be evaluated by using the present model and from the knowledge of results obtained in experiments, by adopting the following procedure.

1. Based on cross-sectional dimensions adopted and the material properties being given or assumed, the quantity $M_{n}$ is determined.

2. $M_{\max }$ is obtained in a straightforward manner from the information regarding $P_{\max }$ obtained through experiments and hence the ratio $M_{\max } / M_{n}$.

3. $E^{*}$ is then directly obtained from Fig. 5 .

Having determined $E^{*}$ corresponding to any practical situation, $\Delta$ is obtained from (5.3) and hence $L_{p_{\max }}$. From the knowledge of $L_{p_{\max }}$, other nonlinear fracture parameters can easily be evaluated.

\subsection{Crack tip opening displacement [CTOD]}

Crack of given length " $a$ " (Fig. 8(a)) is replaced by a fictitious crack of length " $\left(a+L_{p}\right)$ " and subjected to the same external bending moment $M$ (Fig. 8(b)), as well as to a crack closing force $F$ (Fig. 8(c)), being determined from the knowledge of stress distribution in the fracture process zone, across the uncracked ligament. The force $F$ being eccentric, is replaced by an equivalent system consisting of an axial force $F$ and a moment $\bar{M}$. Superposition of schemes adopted in Figs. 8(b) and (c), results in a fictitious cracked beam (Fig. 8(d)) with crack length as, " $\left(a+L_{p}\right)$ " and subjected to an axial closing force $F$ as well as a moment $M^{*}$, being the resultant of $M$ and $\bar{M}$.

With an assumption regarding the centre of rotation being situated at the end of the fracture process zone, the crack tip opening displacement, $\eta$, can be obtained as,

$$
\eta=\theta L_{p}
$$

in which $\theta=$ local rotation ahead of the crack tip.

$\theta$ is computed according to the procedure described by Okamura et al. [24], as

$$
\theta=-\lambda_{F M} F+\lambda_{M} M^{*}
$$

in which $\lambda_{F M}$ and $\lambda_{M}$ are respectively the compliance coefficients of a cracked beam subjected to an axial force and a moment. The above procedure of determining $\theta$, has also been adopted by Wecharatana and Shah [11] and Carpinteri [20].

Using the expressions for $\lambda_{F M}$ and $\lambda_{M}$ as given in [24], an equation for $\theta$ can be obtained as,

$$
\theta=\frac{142.56\left(1-v^{2}\right)}{E B W^{2}}\left[V_{M}(\xi) M^{*}-0.167(1+2 \xi) V_{F M}(\xi) F W\right] \frac{\xi^{2}}{(1-\xi)^{2}(1+2 \xi)^{2}}
$$

in which $\xi=\left(a+L_{p}\right) / W, v=$ Poisson's ratio, $V_{M}(\xi)$ and $V_{F M}(\xi)$ are the parameters required for the determination of compliance coefficients. Values of $V_{M}(\xi)$ and $V_{F M}(\xi)$ for 


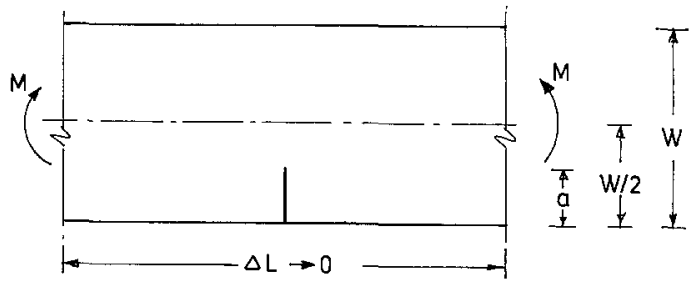

(a)

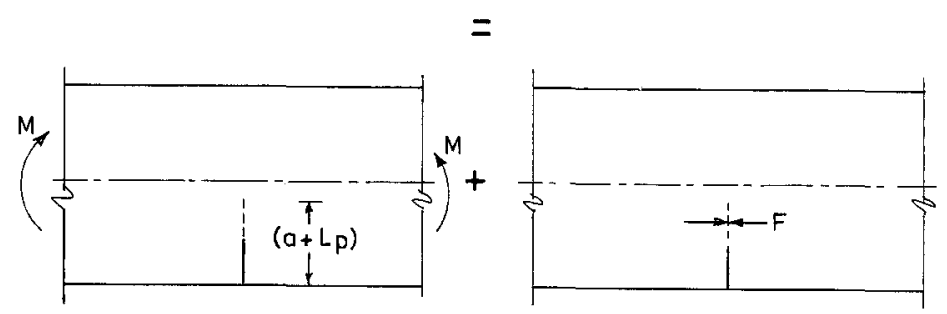

(b)

(c)

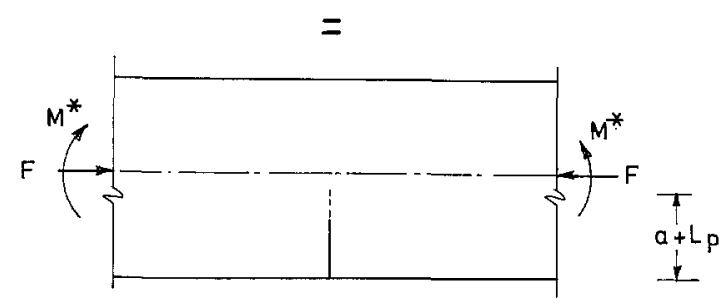

(d)

Fig. 8. Schematic representation of the method adopted, to compute CTOD/CMOD. (a) With actual crack length subjected to bending moment $M$, (b) and (c) are respectively fictitious crack length, subjected to $M$ and crack closing force $F$, (d) resultant section.

various values of $\xi$ as tabulated in [24] are adopted in the present work. This procedure of determining CTOD is adopted mainly because of its simplicity in spite of the availability of more rigorous and accurate methods [25].

\subsection{Crack mouth opening displacement [CMOD]}

This is evaluated in a similar way except that $L_{p}$ in (5.4) is replaced by $\left(L_{p}+a+\right.$ thickness of clip gauge if any). Using the present model, complete P-CMOD curve from the beginning and up to the onset of fracture can be traced. With the following consideration P-CMOD curve even during the physical process of fracture can be obtained. Stress distribution obtained by the present model across the uncracked ligament for post-peak $M / M_{n}$ values less than unity is shown in Fig. 7(b). Such stress distribution seems to be trivial, since compressive stresses cannot exist ahead of the crack tip. Further, based on the proposition that crack propagation proceeds as soon as stress at the crack tip becomes zero, the process zone starts developing into a zone of a macro crack, thus extending the original length of the crack, across which no stress transfer can take place. In view of this, suppressing the compressive stress distribution immediately ahead of the crack tip, one obtains a stress distribution across the uncracked ligament as shown in Fig. 7(c). $M$ is obtained by taking 
moment of tensile forces about the centre of compression (Fig. 7(c)), thus determining the associated value of $P$. CMOD corresponding to this stage is obtained in the usual way.

From (5.6), it is evident that $\theta$ and hence CTOD or CMOD is a function of $E$. With increasing $P$, concrete undergoes progressive damage, thus usage of the same value of $E$ at all stages of loading seems to be inappropriate. From some approximate considerations, a method to estimate the value of $E$ at all stages of loading is suggested below.

\subsection{Effective modulus}

The stress distribution across the uncracked ligament at some stage of loading and at which softening of concrete in tension has occurred is shown in Fig. 3(c). The stress block has been divided into a linear elastic region (AB) and a softening region (BC) with $E$ and $E_{D}$ being respectively the modulii of elasticity. Following the law of mixtures, the effective modulus of elasticity $E_{\text {eff }}$ is obtained as,

$$
E_{\mathrm{eff}}=E(\alpha+\beta)+E_{D}(1-\alpha-\beta)
$$

$E_{D}$ in (5.7) can only represent the average modulus, since modulus of elasticity changes at different locations, with a minimum value, $\bar{E}$ at the location of the crack tip, where obviously maximum damage can occur to a value $E$ at the end of the softening region.

Thus $E_{D}$ is estimated to be the average of $E$ and $\bar{E}$ as,

$$
E_{D}=\frac{E+\bar{E}}{2}
$$

in which $\bar{E}=\sigma / \varepsilon$.

With $\varepsilon_{t}=\sigma_{i} / E, \varepsilon$ can be obtained from the assumption of linear strain distribution across the uncracked ligament as (Fig. 3(d)),

$$
\varepsilon=\frac{\sigma_{t}}{E} \frac{(1-\alpha)}{\beta}
$$

Substituting for $\sigma$ and $\varepsilon$ according to (4.4) and (5.9) in the definition of $\bar{E}$, the following relationship is obtained.

$$
\bar{E}=\left[\frac{\beta-E^{*}(1-\alpha-\beta)}{(1-\alpha)}\right] E
$$

Using (5.8), (5.7) after simplification can be written as,

$$
E_{\mathrm{eff}}=\frac{E}{2}(1+\alpha+\beta)+\frac{\bar{E}}{2}(1-\alpha-\beta)
$$

It is interesting to note from (5.11), that when $\alpha=\beta=0.5, E_{\mathrm{eff}}=E$ is obtained. This seems to be correct since for $P$ corresponding to these values of $\alpha$ and $\beta$, damage to concrete has not yet occurred. 
Having determined $E^{*}$ according to the procedure described in Section 5.1, $\alpha$ and $\beta$ are determined using (4.8) and (4.10), for any stage of loading. $\bar{E}$ can be obtained according to (5.10) and hence an estimate with regard to $E_{\text {eff }}$ can be made from (5.11).

\subsection{Fracture energy}

Several definitions for $G_{F}$ are proposed by different investigators [6-8, 26]. In the present work, definition for $G_{F}$ as proposed by Hillerborg et al. [6] is adopted. Using (4.4) and (5.4), $\sigma$ and $\eta$ can be obtained for all stages of loading. Further, assuming post-peak values for $\sigma$ to vary linearly with $\eta$ right up to fracture, $G_{F}$ is obtained as,

$$
G_{F}=\frac{1}{2} \eta_{f} \sigma_{t}
$$

in which $\eta_{f}$ is the crack tip opening displacement at the onset of fracture.

\section{Analysis of experimental data}

Most of the important experimental data available [23, 27-33] with regard to testing of notched plain concrete beams have been analyzed by using the present model. The implementation of the model requires a priori information regarding the values of $E$ and $\sigma_{t}$ of the concrete mix adopted. The reported values wherever available are adopted, otherwise they are suitably assumed.

\subsection{Discussion of results}

$P$-CMOD curves over the entire range, obtained by using the present model in the cases of beams tested and reported by Jenq and Shah [30], are shown in Figs. (9) to (12). Values of $E$ and $\sigma_{t}$ adopted are indicated. The curves obtained compare very well with those of experimental and theoretical curves [30].

The CTOD corresponding to onset of fracture obtained for all the 80 beam specimens is found to be in the range $8 \mu \mathrm{m}-73 \mu \mathrm{m}$, thus showing a strong dependence on structural size.

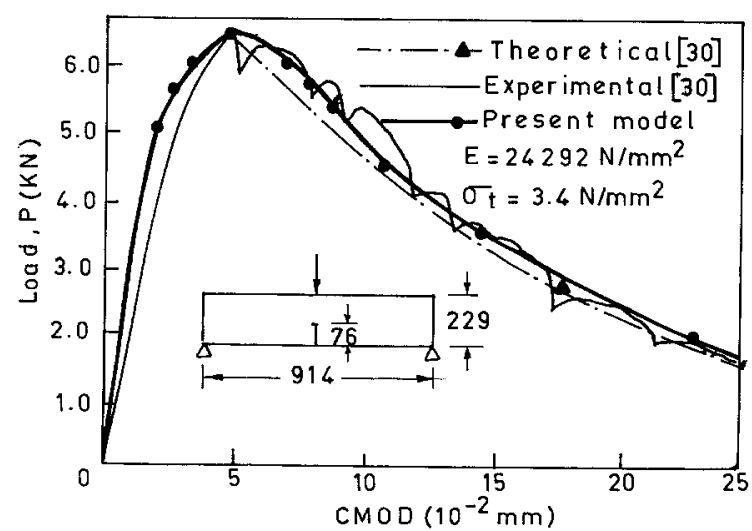

Fig. 9. Comparison of predicted P-CMOD curve, with theoretical and experimental curves [30]. 


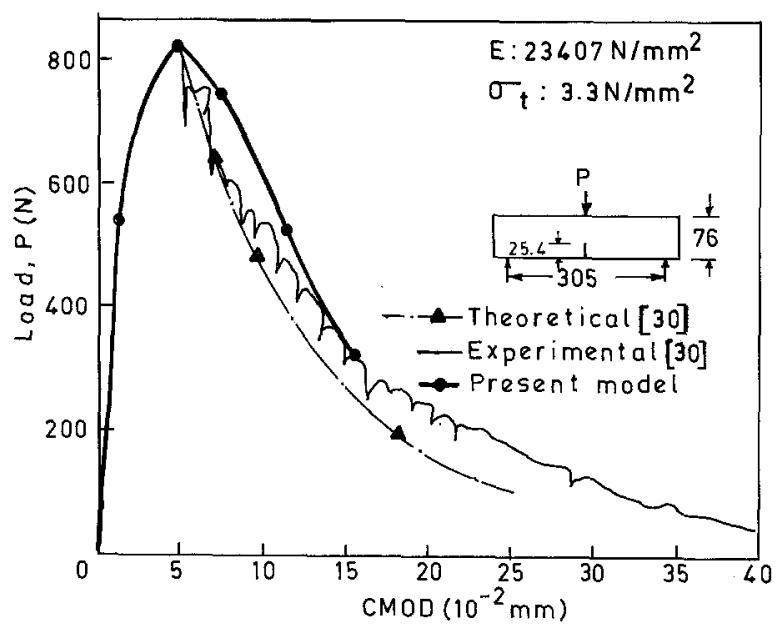

Fig. 10. Comparison of predicted P-CMOD curve, with theoretical and experimental curves [30].

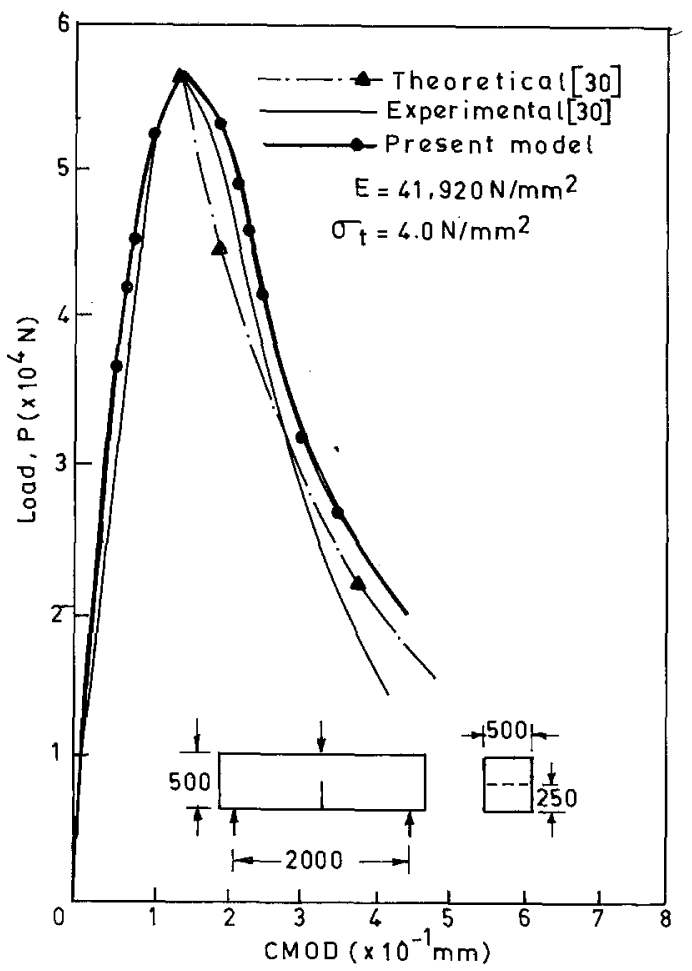

Fig. 11. Comparison of predicted P-CMOD curve, with theoretical and reported experimental curves [30].

Values of CTOD as obtained by different investigators $[15,17]$ at which no stress is transmitted are in the order of $20-40 \mu \mathrm{m}$. Reinhardt et al. [18] have reported the stress-free crack opening to be $160 \mu \mathrm{m}$ for normal weight concrete.

$G_{F}$ in all the cases is found to vary between $10-200 \mathrm{~N} \mathrm{~m}^{-1}$. Values of $G_{F}$ reported by Bazant and $\mathrm{Oh}$ [7] for various fracture test data, are in the range $15-280 \mathrm{~N} \mathrm{~m}^{-1}$.

The value of $L_{p_{\max }}$ is generally found to increase with $(W-a)$ which is in accordance with the observation of Wecharatana and Shah [11]. However, the ratio $L_{p_{\max }} /(W-a)$ is found 


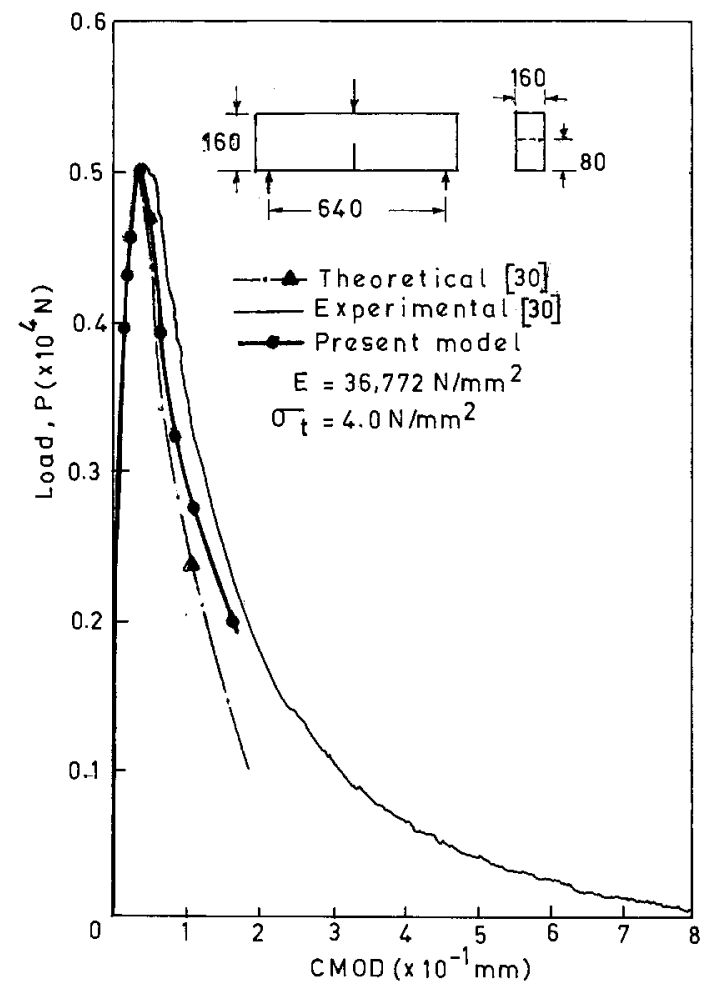

Fig. 12. Comparison of predicted $P$-CMOD curve, with theoretical and reported experimental curves [30].

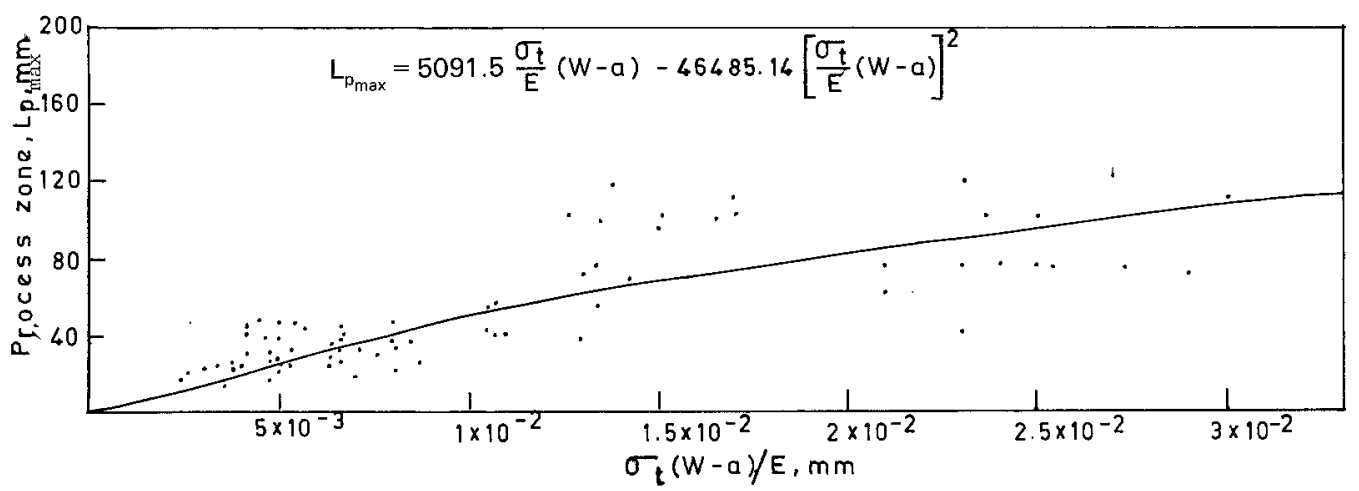

Fig. 13. Prediction of nonlinear process zone length, $L_{p_{\max }}$.

to vary between 0.3 to 0.685 , and to decrease with increase in $(W-a)$. The values of $L_{p_{\max }}$ obtained in all the cases is shown in Fig. 13, as a plot of $L_{p_{\max }}$ versus $\sigma_{i}(W-a) / E$. Since the value of $E^{*}$ is known to depend on structural size, and a relationship connecting them is not available even from the tension test results [15, 19], the regression analysis procedure is adopted in the present work. An empirical relationship for predicting $L_{p_{\max }}$ is obtained as,

$$
L_{p_{\max }}=5091.5 \frac{\sigma_{t}}{E}(W-a)-46485.14\left(\frac{\sigma_{t}}{E}(W-a)\right)^{2}
$$




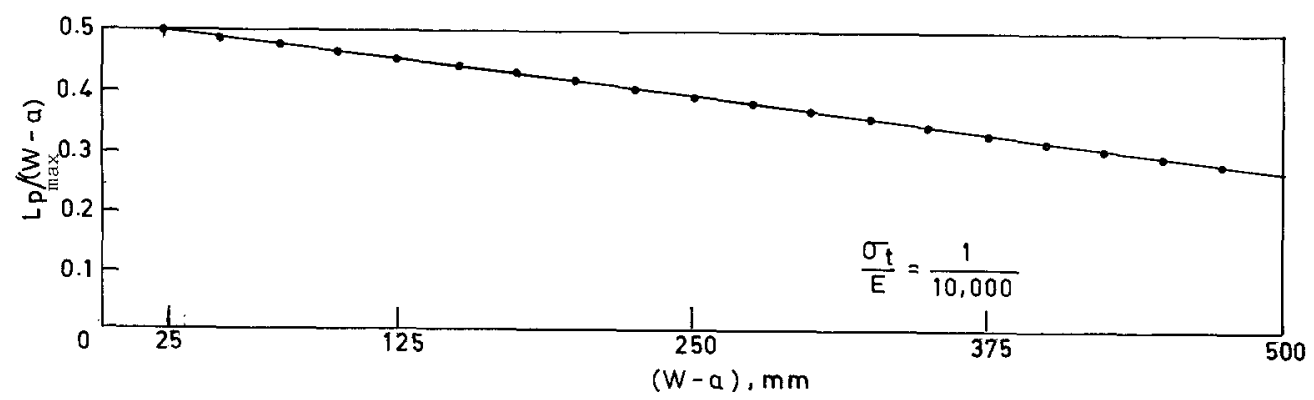

Fig. 14. $L_{P_{\max }} /(W-a)$ versus $(W-a)$.

The predicted values of $L_{p_{\max }}$ according to (6:1), are used to obtain values for $\Delta$ and hence $E^{*}$ according to (5.3). $M_{\max } / M_{n}$ value is then obtained directly from Fig. 5 . With this approach the predicted $M_{\max } / M_{n}$ values in 74 cases out of 80 cases investigated are found to be in close agreement with the experimental values. The discrepancy between the two values is in the range $\pm 0.1 \%- \pm 17 \%$. The remaining six cases where larger discrepancies are obtained are comprised of very small size beams tested by Walsh [28].

For values of $\sigma_{t}(W-a) / E<5 \times 10^{-3}$, prediction of $P_{\max }$ is found to be very sensitive to the value of $L_{p_{\max }}$ given by (6.1). However, for larger values of $\sigma_{l}(W-a) / E$, prediction of $P_{\max }$ is found to be relatively insensitive to the value of $L_{p_{\max }}$, since the change in the values of $M_{\max } / M_{n}$ are very small for larger changes in the value of $E^{*}$, especially for values of $E^{*}$ between $0.5-1$ (Fig. 5). Assuming $\sigma_{t} / E=1 / 10000,(6.1)$ yields $L_{p_{\max }}=0$ for $(W-a)=$ $1.095 \mathrm{~m}$. Since $L_{p_{\max }}=0$, corresponds to brittle fracture, the specimen size as obtained from the present investigation for the brittle fracture to occur should be greater than $1.1 \mathrm{~m}$. This is in good agreement with specimen size requirements obtained by other investigators as summarized in [34]. Values of the ratio $L_{p_{\text {max }}} /(W-a)$, obtained according to (6.1) for various values of $(W-a)$, are shown in Fig. 14, from which it is evident that $L_{p_{\max }} /(W-a)$ decreases with increase in $(W-a)$, a fact which corresponds to the effect of increasing structural size on the fracture behaviour.

Figure 15 shows the influence of increasing values of $(W-a)$ on the value of $E^{*}$ computed according to the present approach for assumed values of $\sigma_{l} / E=1 / 8000,1 / 10000$ and $1 / 12000$. From this figure it can be observed that the value of $E^{*}$ increases with $(W-a)$. However this increase is not proportional throughout. $E^{*}$ is found to increase rapidly for larger values of $(W-a)$.

It can be easily observed that the ratio $M_{\max } / M_{n}$ is identical to the ratio of modulus of rupture (MOR) and $\sigma_{t}$ viz., MOR $/ \sigma_{t}$. Since $M_{\text {max }} / M_{n}$ is found to vary with respect to $E^{*}$ (Fig. 5), and $E^{*}$ is found to be dependent on ( $W-a$ ), (Fig. 15), it is possible to study the variation of MOR $/ \sigma_{t}$ with respect to $(W-a)$. This is indicated in Fig. 16. From this figure it is evident that as $(W-a)$ increases, the value of the ratio MOR $/ \sigma_{t}$ decreases and is found to approach almost unity for $(W-a)$ equal to $1000 \mathrm{~mm}$. From this the influence of structural size of both notched and unnotched beams on the value of MOR can be easily understood. The theoretical prediction of size effect on MOR according to the two-parameter fracture model [13], is also shown in Fig. 16. This curve has been obtained from the suggested value of $Q=12.08$ inches.

From the proposed equation (6.1) for determining $L_{p_{\max }}$ it can be observed that, in the case of beams possessing identical material properties and the same uncracked ligament the 


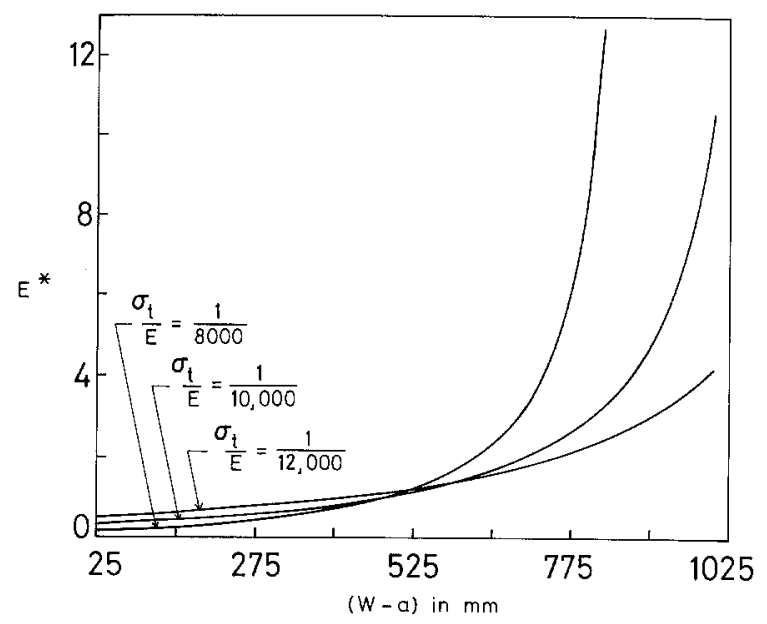

Fig. 15. Influence of $(W-a)$ on $E^{*}$.

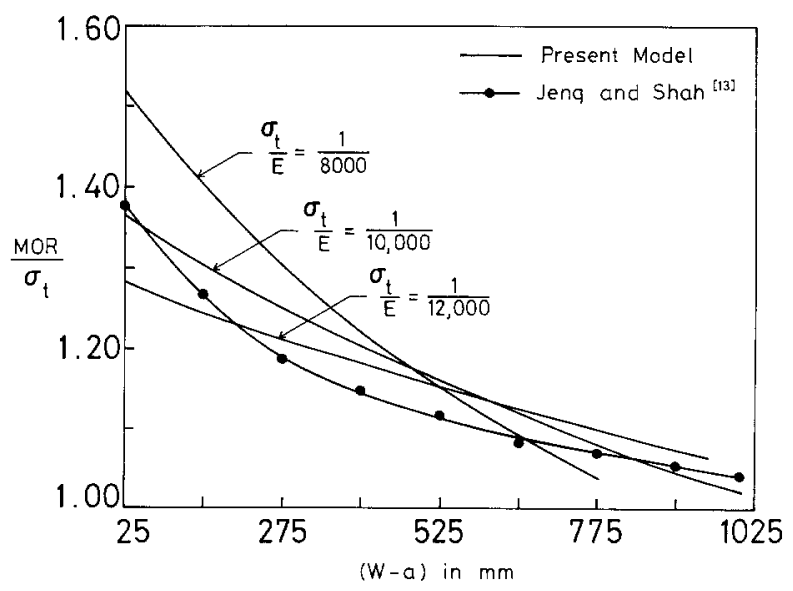

Fig. 16. Influence of $(W-a)$ on $\operatorname{MOR} / \sigma_{t}$.

values of $L_{p_{\text {max }}}$ remain unaltered. The values of $E^{*}$ and $M_{\max } / M_{n}$ will also be the same. Hence, in the case of beams possessing the same material properties, span, breadth, and uncracked ligament, identical values for $P_{\max }$ are obtained irrespective of the overall depth $W$ of the beam. This observation is verified experimentally by performing several tests on notched beam specimens [35]. In these tests the material properties, span, breadth and the uncracked ligament are maintained to be the same while the overall depth of the beams is varied. The indicated values of $P_{\max }$ are also corrected for self weight and additional loads acting on the beam specimens. The experimental results indicate that $P_{\max }$ of the smaller beams is 5 percent more than the $P_{\max }$ for larger beams, although the uncracked ligament is the same in both cases. This observation requires further experimental confirmation. The discrepancy between the predicted values of $P_{\max }$ using the present approach and the test results is again found to be within 10 percent, which is acceptable in a material like concrete. 


\section{Comparison of prediction of $\boldsymbol{P}_{\max }$ from different theoretical models}

Several theoretical models namely, fictitious crack model (FCM) [6], blunt crack band model (BCM) [7], modified fictitious crack model (MFCM) [8] and two parameter fracture model (TPEM) [13] have been proposed so far to analyse the fracture behaviour of plain concrete beams. The primary objective of all these models including the present one is to predict $P_{\max }$. Hence it is interesting to compare the values of $P_{\max }$ predicted according to different models. The results of such a comparative study are summarized in the following paragraphs.

Nallathambi et al. [8] have compared the prediction of $P_{\max }$ by using different numerical models for the case of a beam tested by them. FCM, BCM, and MFCM are found to overestimate $P_{\max }$ by $28.6 \%, 24 \%$ and $22.3 \%$ respectively. Using the values reported in [8] for $\sigma_{t}\left(3.1 \mathrm{~N} \mathrm{~mm}^{-2}\right)$ and $E\left(33000 \mathrm{~N} \mathrm{~mm}^{-2}\right)$, the present model is found to underestimate $P_{\max }$ by 14.54 percent.

Peterson [4] has compared the theoretical results obtained by using FCM with the experimental results. From the test results the average value of $P_{\max }$ is found to be $768.75 \mathrm{~N}$. The theoretical values of $P_{\max }$ according to FCM and corresponding to linear and bi-linear approximations of the post-peak stress versus crack opening displacement diagram are found to be $893.75 \mathrm{~N}(+16.26 \%)$ and $762.5 \mathrm{~N}(-0.8 \%)$. $P_{\max }$ obtained according to the present model for the same values of $\sigma_{t}$ and $E$ is $765.9 \mathrm{~N}(-0.4 \%)$.

The values of $P_{\max }$ in the cases of beams tested by Jenq and Shah [30] are also evaluated according to the present model and TPFM. $P_{\max }$ determined by experiments for large, medium and small beams are $6791 \mathrm{~N}, 2636 \mathrm{~N}$ and $829 \mathrm{~N}$ respectively. The predicted values of $P_{\max }$ according to TPFM are $4522 \mathrm{~N}(-34 \%), 2157 \mathrm{~N}(-18 \%)$ and $577 \mathrm{~N}(-30 \%)$ and according to the present model are $5865 \mathrm{~N}(-14 \%), 2644 \mathrm{~N}(+0.3 \%)$ and $672 \mathrm{~N}(-19 \%)$ respectively. The computation of $P_{\max }$ according to TPFM is based on the average values for the two parameters reported by Jenq and Shah [13], while the values of $P_{\max }$ according to the present model correspond to an assumed value of $\sigma_{t}=3.15 \mathrm{~N} \mathrm{~mm}^{-2}$ (one-eighth of reported value of compressive strength [13]) and the reported value of $E$. It can be observed that the values of $P_{\max }$ predicted according to the present model are in better agreement with the experimental values. It is worth mentioning that by assuming different values for $\sigma_{t}$ and $E$, much better prediction of $P_{\max }$ can be made by using the present model. This can be observed in Figs. 9 and 10. It is to be noted that the values of $E$ indicated in Figs. 9 and 10 are very nearly equal to the value of $E$ calculated from the well known relationship viz., $E=$ $57000 \sqrt{f_{c}^{\prime}}$ psi.

\section{Conclusions}

Based on the results obtained according to the proposed model the following conclusions are made.

1 Softening behaviour of concrete in tension as characterized by the strain softening modulus is a structural property.

2 The ratio of maximum bending moment to net section strength can characterize the fracture behaviour. The value of this ratio equal to unity, indicates brittle fracture, while equal to three refers to plastic collapse. Fracture instability can be expected to occur as soon 
as the stress at the crack tip reduces to zero. Plain concrete beams exhibiting linear strain softening behaviour are found to fracture in the post-peak region at a value of the ratio of bending moment to net section strength equal to unity and not at maximum bending moment itself.

3 Nonlinear fracture parameters such as crack tip opening displacement, crack mouth opening displacement, and fracture energy are highly size dependent.

4 The extent of formation of the fracture process zone across the uncracked ligament depends on the strain softening modulus. The ratio of the length of the fracture process zone to the size of the uncracked ligament decreases with increase in depth.

5 The proposed procedure to predict the maximum load is found to be very sensitive to the value of uniaxial tensile strength for smaller specimens.

6 Nonlinear constitutive law for concrete in compression has very little influence on the fracture behaviour and hence on the maximum load predicted according to linear relationship.

7 The ratio of modulus of rupture to uniaxial tensile strength decreases with the increase in the size of the uncracked ligament and brittle fracture can be expected to occur in the case of plain concrete beams possessing an uncracked ligament depth greater than $1.1 \mathrm{~m}$.

8 The proposed model called Softening Beam Model (SBM) is based on some simple and rational considerations and can completely describe the fracture process from the beginning of formation of the fracture process zone till the onset of fracture instability.

\section{Acknowledgements}

Authors record their thanks to Dr. C. Channakeshava for his helpful discussions during the course of this investigation. Further, the numerical results were obtained using mostly PC/AT which was bought in a research scheme sponsored by University Grants Commission, New Delhi, India. Therefore, authors wish to sincerely thank the UGC for the funds.

\section{References}

1. A. Carpinteri, in Analytical and Experimental Fracture Mechanics, G.C. Sih and M. Mirabile (eds.), Sijthoff and Noordhoff (1981) 785-797.

2. Z.P. Bazant and L. Cedolin, Journal of Engineering Mechanics (ASCE) 105 (1979) 297-315.

3. Z.P. Bazant and L. Cedolin, Journal of Engineering Mechanics (ASCE) 106 (1980) 1287-1306.

4. P.E. Peterson, "Crack Growth and Development of Fracture Zones in Plain Concrete and Similar Materials", Report TVBM-1006, Lund Institute of Technology, Division of Building Materials (1981).

5. A. Carpinteri, Engineering Fracture Mechanics 16 (1982) 467-481.

6. A. Hillerborg, M. Modeer and P.E. Peterson, Cement and Concrete Research 6 (1976) 773-782.

7. Z.P. Bazant and B.H. Oh, Materials and Structures (RILEM) 16 (1983) 155-177.

8. P. Nallathambi and B.L. Karihaloo, Cement and Concrete Research 16 (1986) 373-382.

9. G.C. Sih, in Fracture Mechanics of Concrete: Material Characterization and Testing, A. Carpinteri and A.R. Ingraffea (eds.), Martinus Nijhoff (1984) 1-29.

10. A. Carpinteri, Mechanical Damage and Crack Growth in Concrete, Martinus Nijhoff, Dordrecht (1986).

11. M. Wecharatana and S.P. Shah, Journal of the Engineering Mechanics (ASCE) 109 (1983) 1231-1246.

12. A.S. Kobayashi, N.M. Hawkins, D.B. Barker and B.M. Liaw, in Proceedings NATO Advanced Research Workshop on Application of Fracture Mechanics to Cementitious Composites, S.P. Shah (ed.), (1984) 25-47.

13. Y. Jenq and S.P. Shah, Journal of Engineering Mechanics (ASCE) 111 (1985) 1227-1241. 
14. Z.P. Bazant and P.A. Pfeiffer, ACI Materials Journal, Nov-Dec (1987) 463-480.

15. V.S. Gopalaratnam and S.P. Shah, American Concrete Institute Jounal 3 (1985) 310-323.

16. H.W. Reinhardt, Cement and Concrete Research 15 (1985) 42-52.

17. L. Cedolin, S.D. Poli and I. Iori, Journal of Engineering Mechanics (ASCE) 113 (1987) 431-449.

18. H.W. Reinhardt, H.A.W. Cornelissen and D.A. Hordijk, Journal of Structural Engineering (ASCE) 112 (1986) 2462-2477.

19. F. Ansari, ACI Materials Journal, Nov-Dec (1987) $481-490$.

20. A. Carpinteri, in Fracture Mechanic of Concrete: Structural Application and Numerical Calculation, G.C. Sih and A. DiTommaso (eds.), Martinus Nijhoff (1985) 95-140.

21. A. Carpinteri, Materiaux et Constructions 16 (1983) 85-96.

22. P. Desayi and S. Krishnan, Joumal of the American Concrete Institute 61 (1964) 345-350.

23. H.K. Hillsdorf and W. Brameshuber, in Proceedings NATO Advanced Research Workshop on Application of Fracture Mechanics to Cementitious Composites, S.P. Shah (ed.) (1984) 255-288.

24. H. Okamura and K. Watanabe, Engineering Fracture Mechanics (1975) 531-539.

25. R. Ballarini, S.P. Shah and L.M. Keer, Engineering Fracture Mechanics 20 (1984) 3.

26. P.E. Peterson, Cement and Concrete Research 10 (1980) 79-89.

27. M.F. Kaplan, Journal of the American Concrete Institute, Nov. (1961) 591-609.

28. P.F. Walsh, Indian Concrete Journal 46 (1972) 469-460, 476.

29. O.E. Gjorv, S.I. Sorensen and A. Arenesen, Cement and Concrete Research 7 (1977) 333-344.

30. Y. Jenq and S.P. Shah, in Proceedings NATO Advanced Research Workshop on Application of Fracture Mechanics to Cementitious Composites, S.P. Shah (ed.), (1984) 213-253.

31. P. Nallathambi, B.L. Karihaloo and B.S. Heaton, Cement and Concrete Research 15 (1985) 117-126.

32. P.E. Peterson, Cement and Concrete Research 10 (1980) 91-101.

33. P.M. Catalano and A.R. Ingraffea, "Concrete Fracture: A linear Elastic Fracture Mechanics Approach", report No. 82-1, Department of Structural Engineering, Cornell University (1982).

34. S. Mindess, in Fracture Mechanics of Concrete: Material Characterization and Testing, A. Carpinteri and A.R. Ingraffea (eds.), Martinus Nijhoff (1984).

35. H. Ananthan, Ph.D. thesis, "Fracture Behaviour of Plain and Reinforced Concrete Beams", Department of Civil Engineering, Indian Institute of Science, Bangalore, India (1989).

Résumé. A l'aide d'un modèle unidimensionnel dit "Softening Beam Model" (SBM), on analyse le comportement à la rupture de poutres élancées pleines entaillées ou non, soumises en flexion en trois ou quatre points. Des équations fondamentales d'équilibre sont utilisées pour développer le modèle. On explique l'influence de la taille du composant sur l'altération du mode de rupture en rupture fragile et en effondrement plastique par la distribution par la distribution des contraintes sur le ligament non fissuré lorsque varie le module d'adoucissement. On trouve que la contrainte à l'extrémité de la fissure est nulle est nulle au début de l'instabilité de la rupture. La charge maximum et la charge à la rupture sont trouvées différentes, et on obtient une valeur unique de la charge à la rupture. On montre que la longueur de la zone concernée par le processus de rupture d'pend de la valeur du module d'adoucissement. On calcule également les limites théoriques de longueur de cette zone. Divers paramètres de rupture non linéaire sont calculés pour une large gamme d'éprouvettes en poutres reprises dans la littérature; on trouve qu'il existe une bonne concordance avec les résultats expérimentaux et théoriques. On démontre qu'en suivant une procédure simple on peut obtenir avec une bonne précision la courbe reliant les portions avant et après le pic de sollicitation en fonction du COD de la fissure. En outre, on développe une procédure simple pour calculer la charge maximum. Les valeurs prédites sont en bon accord avec les valeurs expérimentales. Le modèle SBM proposé est très simple et est basé sur des considérations rationnelles. Il est susceptible de décrire complètement le processus de rupture depuis le début de la formation de la zone intéressée jusqu'à l'amorçage de la rupture instable. 